\title{
Evidence for tissue-resident mesenchymal stem cells in human adult lung from studies of transplanted allografts
}

\author{
Vibha N. Lama, ${ }^{1}$ Lisa Smith, ${ }^{2}$ Linda Badri, ${ }^{1}$ Andrew Flint, ${ }^{2}$ Adin-Cristian Andrei, ${ }^{3}$ Susan Murray, ${ }^{3}$
} Zhuo Wang, ${ }^{4}$ Hui Liao, ${ }^{1}$ Galen B. Toews, ${ }^{1}$ Paul H. Krebsbach, ${ }^{4}$ Marc Peters-Golden, ${ }^{1}$ David J. Pinsky, ${ }^{1}$ Fernando J. Martinez, ${ }^{1}$ and Victor J. Thannickal' ${ }^{1}$

${ }^{1}$ Department of Internal Medicine, Division of Pulmonary and Critical Care Medicine, and 2Department of Pathology, University of Michigan Health System, Ann Arbor, Michigan, USA. ${ }^{3}$ Department of Biostatistics, University of Michigan School of Public Health, Ann Arbor, Michigan, USA. ${ }^{4}$ University of Michigan School of Dentistry, Ann Arbor, Michigan, USA.

\begin{abstract}
The origin and turnover of connective tissue cells in adult human organs, including the lung, are not well understood. Here, studies of cells derived from human lung allografts demonstrate the presence of a multipotent mesenchymal cell population, which is locally resident in the human adult lung and has extended life span in vivo. Examination of plastic-adherent cell populations in bronchoalveolar lavage samples obtained from 76 human lung transplant recipients revealed clonal proliferation of fibroblast-like cells in $62 \%$ (106 of 172) of samples. Immunophenotyping of these isolated cells demonstrated expression of vimentin and prolyl-4-hydroxylase, indicating a mesenchymal phenotype. Multiparametric flow cytometric analyses revealed expression of cell-surface proteins, CD73, CD90, and CD105, commonly found on mesenchymal stem cells (MSCs). Hematopoietic lineage markers CD14, CD34, and CD45 were absent. Multipotency of these cells was demonstrated by their capacity to differentiate into adipocytes, chondrocytes, and osteocytes. Cytogenetic analysis of cells from 7 sex-mismatched lung transplant recipients harvested up to 11 years after transplant revealed that $97.2 \% \pm 2.1 \%$ expressed the sex genotype of the donor. The presence of MSCs of donor sex identity in lung allografts even years after transplantation provides what we believe to be the first evidence for connective tissue cell progenitors that reside locally within a postnatal, nonhematopoietic organ.
\end{abstract}

\section{Introduction}

Mesenchymal stem cells (MSCs) are multipotent progenitor cells with the capacity to differentiate into connective tissue cells $(1,2)$. Bone marrow, an enriched reservoir of MSCs, is believed to be the main source for these precursor cells that populate other adult tissues $(3,4)$. MSCs can be isolated from circulating blood (5), as well as from diverse nonhematopoietic tissues such as synovium (6), adipose tissue (7), trabecular bone (8), dermis (9), dental pulp (10), and the lung (11). It remains unknown whether MSCs isolated from these marrow-distant tissues reside and self-renew locally within that tissue/organ system or whether they are recruited from the circulation/bone marrow during postnatal adult life.

The lung is continuously exposed to a variety of potentially injurious pathogens and noxious agents, necessitating cellular turnover and renewal. Although MSCs have been detected in the lung (11), their origin(s) remain unknown, and an evolving paradigm predicts that mesenchymal cells participating in lung repair derive from the bone marrow (12-14). There have been no reports of MSCs that have been conclusively demonstrated to be of tissuespecific origin in any adult organ system, including the lung.

The transplanted lung is subject to a multitude of insults, including ischemia and reperfusion, immunologic injury, and microbial infections, triggering host tissue repair and regenerative

Nonstandard abbreviations used: aP2, adipocyte fatty acid binding protein 2; BAL, bronchoalveolar lavage; BOS, bronchiolitis obliterans syndrome; CFU-F, CFU-fibroblast; FABP4, fatty acid binding protein 4, adipocyte; MSC, mesenchymal stem cell. Conflict of interest: The authors have declared that no conflict of interest exists. Citation for this article: J. Clin. Invest. 117:989-996 (2007). doi:10.1172/JCI29713. responses (15). Mesenchymal cell accumulation/activation and tissue remodeling are important in the pathogenesis of chronic graft failure in transplanted solid organs. In the lung, chronic graft failure manifests as airflow obstruction referred to as bronchiolitis obliterans syndrome (BOS) (16). The origins of mesenchymal cells that participate in normal and abnormal repair responses in the human adult lung remain unclear. In this study, we demonstrate the isolation of multipotent MSCs from the lower respiratory tract of human lung transplant recipients. Additionally, studies in sexmismatched lung allografts were conducted to determine whether these cells originate from the engrafted lung or from the host circulation/bone marrow.

\section{Results}

Patient characteristics. Between May 2004 and December 2005, 172 bronchoalveolar lavage (BAL) fluid samples from 76 lung transplant recipients were collected and analyzed at the University of Michigan Medical Center. This patient population included 42 females and 34 males with a mean age of 51 years (range, 23-64 years). Indications for transplantation included emphysema $(n=39)$, idiopathic pulmonary fibrosis $(n=16)$, cystic fibrosis $(n=10)$, bronchiectasis $(n=3)$, lymphangioleiomyomatosis $(n=2)$, primary pulmonary hypertension $(n=2)$, sarcoidosis $(n=2)$, and other diagnoses $(n=2)$. One hundred thirty BALs were performed during routine surveillance to rule out acute rejection or infection, and 42 BALs were performed for other clinical indications such as unexplained dyspnea or decline in lung function.

Isolation of plastic-adherent fibroblastoid cells from BAL fluid. MSCs have been recognized to derive from single-cell suspensions of 
A

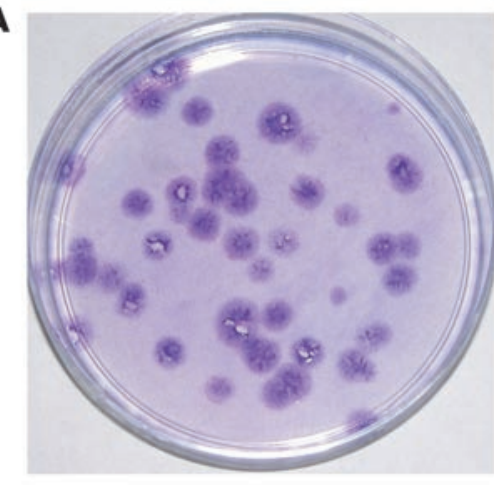

B

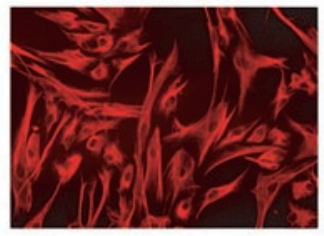

Prolyl-4-hydroxylase

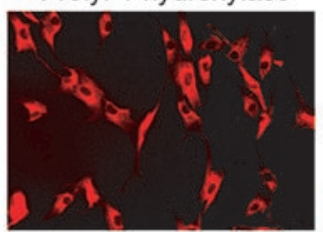

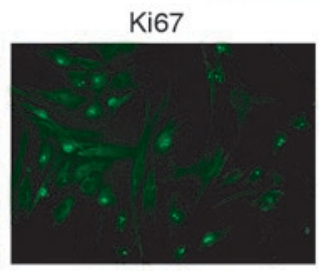

Ki67

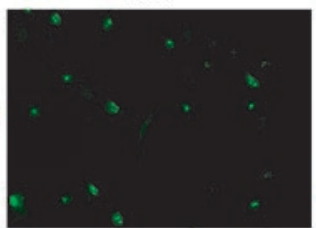

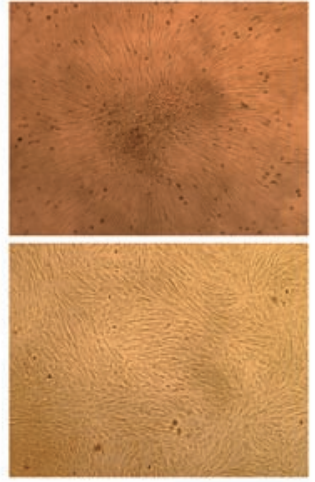

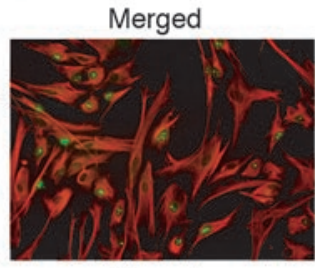

Merged

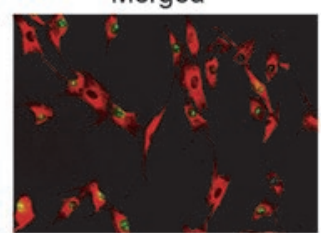

\section{Figure 1}

Isolation and characterization of mesenchymal cells from BAL of lung transplants. (A) Mononuclear cells obtained from BAL of lung allografts were plated and maintained in culture. Giemsa staining at day 10 identified the CFU-Fs (left). The top right panel shows an individual CFU-F (original magnification, $\times 10$ ). On subsequent trypsinization and culturing, a homogeneous population of mesenchymal cells was obtained by passage 2 (bottom right). (B) Immunofluorescence staining of mesenchymal cells at passage 3 demonstrated expression of vimentin (an intermediate filament protein), prolyl-4hydroxylase (a collagen cross-linking enzyme), and Ki67 (a marker of proliferation). Merged images (right) demonstrated that the majority of vimentin- and prolyl-4-hydroxylaseexpressing mesenchymal cells were actively proliferating.

strated expression of vimentin (an intermediate filament protein) and prolyl-4-hydroxylase (an enzyme that catalyzes posttranslational cross-linking of collagen), proteins typically associated with mesenchymal cells (Figure 1B). The high proliferative capacity of these cells was confirmed by staining for Ki67 protein (Figure 1B).

Immunophenotyping for surface antigens by flow cytometry was performed on BAL-derived mesenchymal cells to further characterize this cell population. These cells strongly expressed CD73 (SH3/SH4), CD90 (Thy-1), and CD105 (SH2) (Figure 2), as previously described for bone marrow-derived MSCs $(1,20)$. Furthermore, these cells were negative for the hematopoietic lineage markers

bone marrow by the selective growth of plastic-adherent fibroblastlike cell colonies in liquid culture medium (17). Such a colony of adherent marrow stromal cells, each derived from a single precursor cell, is termed a CFU-fibroblast (CFU-F) (18). To determine whether MSC-like cells can be isolated from the lung, the cellular components of BAL fluid were examined. Distinct fibroblastoid colonies were observed at a mean time interval of 14 days (range, 7-21 days) after initial plating of BAL cells (Figure 1A). The number of CFU-Fs formed per $2 \times 10^{6}$ mononuclear cells plated varied among samples (mean, 20; range, 0-74). CFU-Fs were identified in $62 \%$ (106 of 172) of the BAL samples obtained from lung transplant recipients. These CFU-Fs were expanded in culture by subsequent trypsinization and serial passaging (Figure 1A).

The influence of clinical variables on the ability to isolate CFU-Fs from the BAL fluid was analyzed using generalized estimating equations (19), a standard statistical technique for analysis of longitudinal data (Table 1). These data indicated that neither age, sex, indication for transplantation or BAL, culture positivity, histological diagnosis of rejection or bronchiolitis obliterans, nor physiological evidence for BOS predict ability to successfully isolate CFU-Fs. The only clinical variable that negatively predicted ability to isolate CFU-Fs was increasing time from transplantation to BAL. This was demonstrated by both univariate (odds ratio for each additional year of time from transplant to BAL, 0.86; 95\% CI, $0.77-0.96 ; P=0.007$ ) and multivariate analyses (odds ratio, 0.78; $95 \% \mathrm{CI}, 0.65-0.95 ; P=0.015)$, after adjusting for all other recipient and BAL variables shown in Table 1 .

Phenotypic characterization of BAL-derived CFU-Fs. CFU-Fs initially identified from the BAL were expanded in ex vivo cell culture without morphological evidence of cellular senescence. Immunofluorescence studies of these BAL-derived cells demonCD14, CD34, and CD45 (Figure 2). HLA typing revealed that these cells express HLA-1, but not HLA-DR or HLA-DQ (data not shown).

Multipotential differentiation of BAL-derived mesenchymal cells. As the major defining characteristic of MSCs is their ability to differentiate into multiple mesenchymal lineages, we determined the ability of BAL-derived mesenchymal cells to differentiate into specific connective tissue cells. Mesenchymal cell lines from 15 patients (at passages 2-6) were studied under defined culture conditions to induce osteocytic, adipocytic, and chondrocytic differentiation (as described in Methods). The ability of BAL-derived mesenchymal cells to differentiate into osteocytes was demonstrated by staining for extracellular mineralization in cells following osteogenic stimulation $(1,10,11)$ (Figure 3A, left panels). Osteocytic differentiation was seen in all 15 cell lines tested and was absent in control untreated cells. Adipocytic differentiation was demonstrated in 14 of 15 cell lines stimulated with insulin and other adipogenic stimuli $(1,10,11)$ by accumulation of lipid-rich vacuoles that stained positively with oil red $\mathrm{O}$ (Figure 3A, center panels). No oil red $\mathrm{O}$ staining was seen in the control cells. Pelleted micromasses of cells treated with TGF- $\beta_{3}$ (1) underwent chondrocytic differentiation, as evidenced by the presence of the extracellular matrix proteoglycan aggrecan in all treated cell lines (Figure 3A, right panels). These studies suggest that BAL-derived mesenchymal cells are capable of differentiating into multiple connective tissue cell lineages.

To determine whether BAL-derived mesenchymal cells contain early uncommitted MSCs capable of differentiating into all 3 mesenchymal cell lineages (as opposed to mixtures of more committed progenitor cells), single colonies were isolated and expanded in culture prior to assays of multipotential differentiation. Ten cell lines derived from individual CFU-Fs were tested; all colonies were capable of undergoing differentiation into osteocytic and chondro- 
Table 1

\begin{tabular}{|c|c|c|c|c|}
\hline Variable & $\begin{array}{l}\text { CFU-Fs noted in } \\
\text { BAL fluid }(n=106)\end{array}$ & $\begin{array}{l}\text { No CFU-Fs noted in } \\
\text { BAL fluid }(n=66)\end{array}$ & $\begin{array}{l}\text { Odds } \\
\text { ratio }\end{array}$ & $P^{B}$ \\
\hline Age $(y r)$ & $51 \pm 11$ & $48 \pm 12$ & 1.90 & 0.78 \\
\hline $\begin{array}{l}\text { Sex (male/female) } \\
\quad \text { Indication for transplant }\end{array}$ & \multicolumn{3}{|c|}{ Indication for transplant } & 0.45 \\
\hline Emphysema & 54 & 27 & 1.82 & 0.25 \\
\hline Idiopathic pulmonary fibrc & rosis & 14 & 1.20 & 0.75 \\
\hline Cystic fibrosis & 11 & 13 & 1.04 & 0.95 \\
\hline Others & 15 & 12 & $1.00^{C}$ & $N A^{C}$ \\
\hline \multicolumn{4}{|l|}{ Indication for BAL } & 0.02 \\
\hline Symptoms & 23 & 19 & 1.35 & 0.66 \\
\hline Surveillance & 83 & 47 & $1.00^{C}$ & $N A^{C}$ \\
\hline \multicolumn{5}{|l|}{ Microbiological culture } \\
\hline Bacterial & 9 & 13 & 0.38 & 0.10 \\
\hline CMV & 10 & 5 & 1.25 & 0.76 \\
\hline No growth & 83 & 46 & $1.00^{C}$ & $N A^{C}$ \\
\hline \multicolumn{5}{|l|}{ Histological diagnosis } \\
\hline Acute rejection & 16 & 6 & 1.74 & 0.31 \\
\hline Bronchiolitis obliterans & 4 & 4 & 0.77 & 0.77 \\
\hline Lymphocytic bronchitis & 3 & 1 & 3.14 & 0.25 \\
\hline Other pathologies & 7 & 3 & 2.34 & 0.41 \\
\hline Normal & 76 & 52 & $1.00^{C}$ & $N A^{C}$ \\
\hline Presence of BOS & 17 & 14 & 1.42 & 0.67 \\
\hline
\end{tabular}

All values are number unless otherwise indicated. ${ }^{A}$ Odd ratios represent multivariate generalized estimating equation models accounting for correlation within patients, adjusted for all other factors shown. Odds ratios for continuous factors reflect 1 unit increase in factor. ${ }^{B} P$ values generated from multivariate generalized estimating equation models accounting for correlation within patients. Multivariate $P$ values adjust for all other factors shown. ${ }^{C}$ Reference population.
Clinical variables influencing the identification of CFU-Fs in BAL samples

phosphatase expression levels were increased significantly in response to adipogenic and osteogenic differentiation media, respectively (data not shown). Baseline expression of these genes in BAL-derived MSCs was compared with that in bone marrow-derived MSCs by real-time PCR. No significant difference in baseline expression of adipocytic markers (PPAR $\gamma$, FABP4), chondrocytic markers (aggrecan), or alkaline phosphatase was seen between BAL- and bone marrowderived MSCs. However, 167-fold greater expression of osteopontin was noted in the bone marrow-derived MSCs compared with BAL-derived MSCs $(P=0.007)$.

The ability of individual CFU-Fs from the BAL fluid to each give rise to multiple mesenchymal lineages supports the presence of uncommitted MSCs in the airway-alveolar compartment of lung transplant recipients.

Sex chromosome analyses by FISH of BALderived MSCs. MSCs isolated from nonhematopoietic tissues may either derive from the bone marrow/circulation or reside locally within the tissue/organ. To distinguish the tissue of origin of these BALderived MSCs, the recipient versus donor sex chromosome status of cells from 7 sexmismatched transplant recipients was studied. Five hundred cells from each patient were analyzed at passage 3 by FISH for $\mathrm{X}$ (red) and Y (green) chromosomes (Figure 4;

cytic lineages, and 6 of 10 demonstrated adipocytic differentiation. Additional molecular characterization of these differentiated cells was performed by mRNA expression analysis of lineage-specific markers. Upregulated expression of PPAR $\gamma 2$ and adipocyte fatty acid binding protein 2 (aP2) (adipogenic transcription factors), types II and IX collagen (chondrocytic markers), and osteopontin (osteocytic marker) was demonstrated under their respective differentiation-inducing conditions by RT-PCR (Figure 3B). Quantification of upregulated expression for osteopontin, PPAR $\gamma$, and aggrecan, indicative of osteogenic, adipogenic, and chondrogenic differentiation, respectively, was demonstrated by real-time PCR (Figure 3C). Similarly, fatty acid binding protein 4 , adipocyte (FABP4) and alkaline

\section{Figure 2}

Immunophenotyping of mesenchymal cells isolated from BAL fluid of lung transplant recipients by flow cytometric analysis. Lung mesenchymal cells were isolated at passage 2 and immunostained for cell-surface markers with specific mAbs. These cells were predominantly positive for CD73, CD90, and CD105 (upper panels) and uniformly negative for the hematopoietic lineage markers CD14, CD34, and CD45 (bottom panels). All histograms show specific mAbs in purple and control isotype-specific IgGs in green. The percentage of positive cells relative to total cells analyzed (mean $\pm S D$ ) is shown above each histogram.
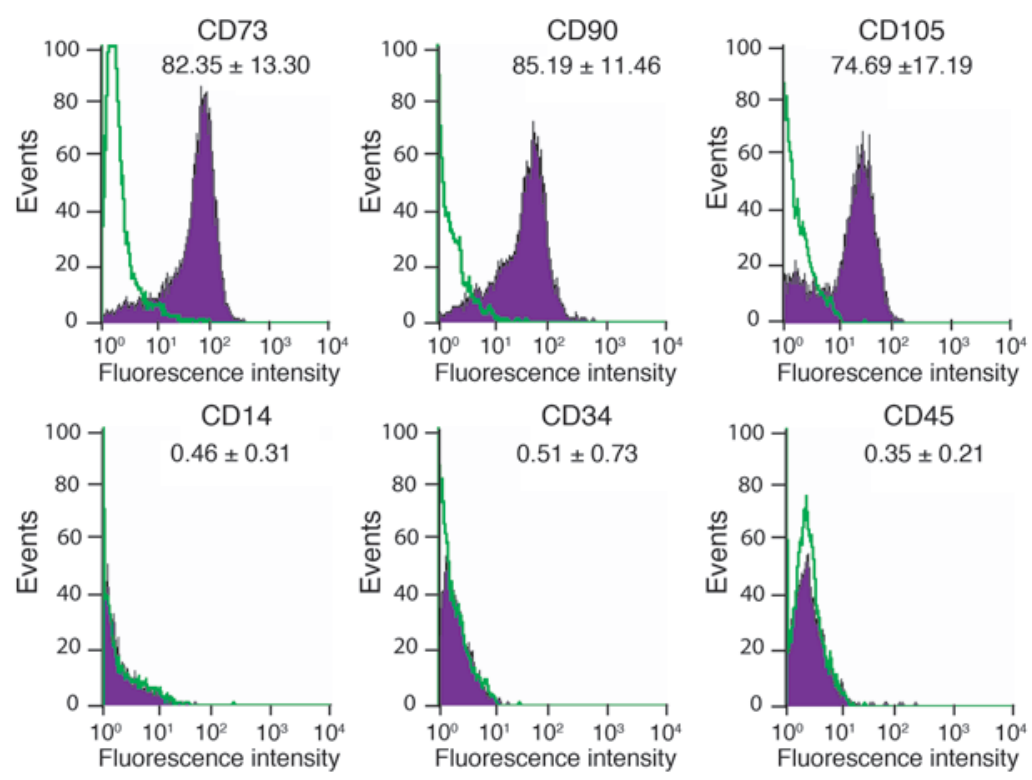
A
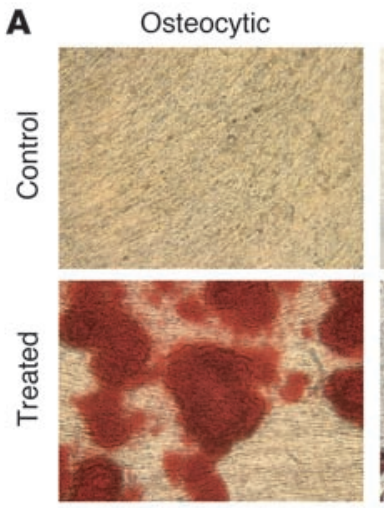

B
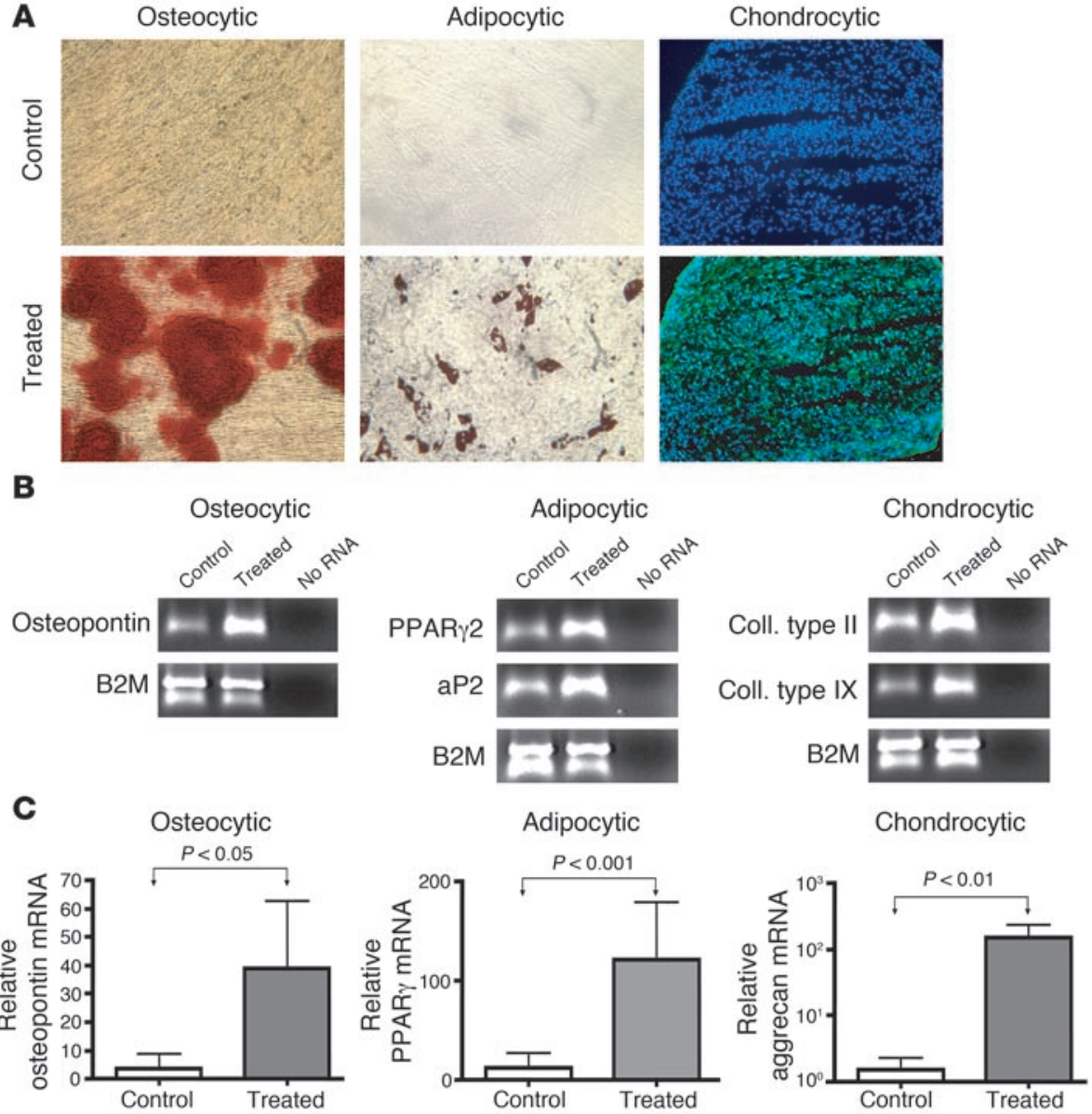

Figure 3

Mesenchymal cells isolated from BAL fluid of lung transplant recipients differentiate to multiple connective tissue lineages. (A) Cultured mesenchymal cells from 15 BAL samples and 10 individual CFU-Fs were investigated for in vitro multilineage differentiation capacity. Osteocytic differentiation was indicated by calcium deposition as demonstrated by alizarin red staining (red) in treated cells (bottom left). This was absent in control untreated cells (top left). Accumulation of lipid droplets (indicating adipocytic differentiation) was demonstrated by staining with oil red $\mathrm{O}$ in treated cells (bottom center; untreated control is shown in top center). Immunofluorescence staining with anti-human aggrecan polyclonal antibody demonstrates the presence of the extracellular matrix proteoglycan aggrecan, indicative of chondrocytic differentiation in treated cells (bottom right panel; aggrecan-FITC: green; DAPI: blue). A contiguous section stained with control IgG antibody is shown (top right). (B) RT-PCR was performed to analyze the expression of mRNAs specifically related to adipogenic, chondrogenic, and osteogenic activity under inductive culture conditions. Ten cell lines generated from individual CFU-Fs were cultured in either control or differentiation-inducing conditions. Expression of PPAR $\gamma$ and aP2 (indicative of adipogenic activity), type II collagen and type IX collagen (Coll. type II and IX; indicative of chondrogenic activity), and osteopontin (indicative of osteogenic activity) was upregulated in treated cells. Lanes demonstrate RNA isolated from untreated cells, RNA isolated from cells undergoing differentiation, and RNase-Free Distilled Water (UltraPure; Invitrogen), respectively. B2M, $\beta_{2}$-microglobulin. (C) Real-time PCR provided a quantitative analysis of increased relative expression of mRNAs specifically related to adipogenic, chondrogenic, and osteogenic activity under inductive culture conditions.

the BAL fluid of these patients exhibited the XY chromosomal status of the donor. No cells ( 0 of 500) of recipient origin were noted in 5 of 7 cases. In the other 2 cases, only 1 of 500 and 8 of 500 cells studied were of recipient origin. This indicates that MSCs isolated from BAL fluid of lung allografts are locally resident within the adult lung and not derived from an extrapulmonary or bone marrow MSC population.
To determine the effect of time following transplantation on donor versus recipient origin of MSCs, sex chromosome assignment data were stratified according to time out from the transplantation procedure. Cells were obtained for study from patients at early ( $\leq 3$ months in patients $1-3$ ) and late $(3.1,3.9,7.4$, and 11.5 years in patients $4,5,6$, and 7 , respectively; Table 2 ) time points after lung transplantation. In both these cohorts of patients, BALderived MSCs consistently demonstrated the donor sex genotype. These data illustrate the presence of donor-derived MSCs long after lung transplantation, suggesting their longevity or capacity for self-renewal in vivo.

Cytokine/chemokine gene expression profile of lung-resident MSCs versus bone marrow-derived MSCs. The best-characterized source of MSCs in humans is the bone marrow $(1,18,20)$. To determine whether cytokine/chemokine gene expression profiles in MSCs resident in the lung are distinct from those derived from the bone marrow, we compared the transcriptome of MSCs from these sources by Affymetrix analysis. The majority of genes previously demonstrated to be highly upregulated in MSCs (21-23) were found to be expressed to a similar degree in both BAL- and bone marrow-derived MSCs (Supplemental Table 1, genes 1-19; supplemental material available online with this article; doi:10.1172/JCI29713DS1). However, several chemokines (IL-8, CXCL1, CXCL2, CXCL6), genes associated with angiogenesis (encoding IL- $1 \alpha$, IL-8, VEGF, FGF2, and TGF- $\beta 2$ ), and genes associated with hematopoiesis (encoding leukemia inhibitory factor, CSF1-macrophage, CSF2 granulocyte-macrophage, and IL-6) were more highly expressed in bone marrowderived MSCs than BAL-derived MSCs. Affymetrix data also confirmed realtime PCR data indicating increased expression of osteopontin, a marker for osteogenesis and an important factor for hematopoiesis, in bone marrowderived MSCs compared with BALderived MSCs. The data suggest that MSCs derived from these different tissue environments reflect distinct gene expression profiles consistent with their origin and functional roles.

Isolation of MSCs from BAL fluid obtained from nontransplant lungs. To determine whether MSCs can be isolated from BAL fluid in the absence of lung transplantation, BAL samples were obtained from healthy volunteers. Adherent mesenchymal cells were obtained from 
Donor: male

Recipient: female
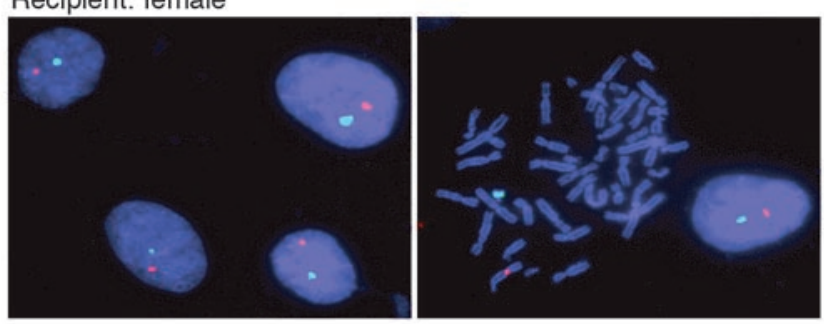

Donor: female

Recipient: male
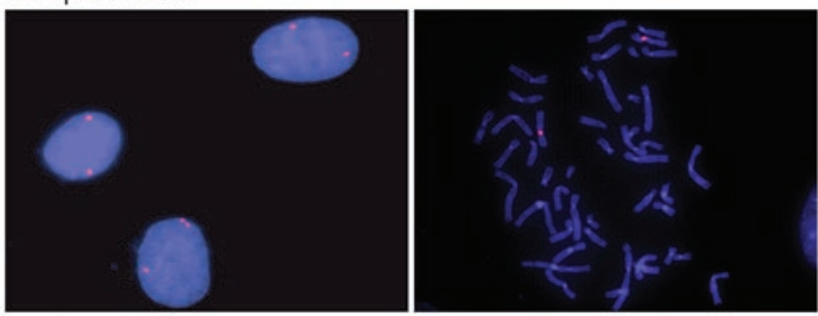

5 of 15 samples processed in a manner identical to that described for lung transplant recipients. The ability of mesenchymal cells obtained from normal subjects to undergo multilineage differentiation in response to various differentiation-inducing conditions was tested in 3 of these cell lines. Microscopy revealed the presence of fat globules and calcium accumulation in response to adipocytic and osteocytic differentiation conditions, respectively; in addition quantitative real-time PCR assays revealed a 12-fold increase in FABP4 activity ( $P=0.003$; adipocytic differentiation) and a 9-fold increase in alkaline phosphatase activity $(P=0.02$; osteocytic differentiation). Each of the BAL-derived mesenchymal cells from these healthy subjects stained positively for chondrocytic markers in response to TGF- $\beta_{3}$, similar to what was observed in samples from lung transplant recipients (Figure 3A). These data demonstrate that mesenchymal cells with multilineage differentiation potential can be isolated from BAL fluid obtained from human lungs in the absence of an allogeneic stimulus. However, our preliminary results suggest that the ability to recover these cells in normal volunteers may be lower than that seen in the lung transplant population.

\section{Discussion}

In this study, we demonstrate the ability to isolate fibroblast-like cells from the lower respiratory tract of lung transplant recipients

\section{Figure 4}

MSCs isolated from BAL fluid of transplanted lungs are of donor sex genotype. FISH of mesenchymal cells isolated from BAL fluid of sexmismatched lung transplant recipients was performed using CEP $X$ Spectrum Orange and $Y$ Spectrum Green DNA probes to distinguish cells of donor versus recipient origin. The top row shows the male sex chromosome status of mesenchymal cells obtained from BAL of a male donor lung transplanted into a female host. Red signal indicates $\mathrm{X}$ chromosome, and green signal indicates $\mathrm{Y}$ chromosome. Cells were scored in interphase (left). A cell in metaphase demonstrating specificity of the DNA probes is shown on the right. Bottom panels demonstrate the female sex chromosome status of mesenchymal cells obtained from BAL of a female donor lung transplanted into a male recipient (left: interphase cells; right: representative cell in metaphase).

with a minimally invasive procedure commonly used in clinical practice, bronchoscopy with BAL. BAL-derived mesenchymal cells exhibited several characteristics typical of classical bone marrowderived MSCs $(1,18,20)$. First, they can be isolated by adherence purification on tissue culture plastic and form distinct CFU-Fs in cell culture; second, these cells express surface proteins typically associated with MSCs but do not express hematopoietic stem cell markers; third, individual colonies possess multipotent differentiation capacity and give rise to adipocytes, chondrocytes, and osteocytes. Importantly, cytogenetic analyses in sex-mismatched recipients demonstrated that these BAL-derived MSCs were of donor sex chromosome karyotype, even when analyzed a number of years after lung transplantation. This is the first study to our knowledge to demonstrate that lung tissue-specific MSCs are present in the human adult lung. The long-term survival of these cells - for more than a decade in transplanted lung allografts - supports the concept of the presence of organ-specific MSC populations that likely serve as a reservoir for connective tissue cells in nonhematopoietic organ systems.

There are a growing number of reports of putative MSC-like cells isolated from various nonhematopoietic organs, including the lung (6-11). Importantly, however, no human or animal studies have definitively determined whether these cells are tissue

\section{Table 2}

Sex chromosome status of MSCs isolated from sex-mismatched recipients

\begin{tabular}{lccccccccc} 
Case & Time from & BOS & Recipient & Donor & \multicolumn{5}{c}{ No. of MSCs (500 cells scored/patient) with donor sex chromosome status } \\
no. & transplant (d) & & seX & seX & XY & XX & X & XXXX & XXY \\
1 & 24 & No & XY & XX & 0 & 487 & 2 & 11 & 0 \\
2 & 87 & No & XX & XY & 472 & 0 & 0 & 0 & 0 \\
3 & 91 & No & XX & XY & 483 & 0 & 0 & 0 & 0 \\
4 & 1,129 & Yes & XX & XY & 500 & 0 & 0 & 0 & 0 \\
5 & 1,424 & Yes & XX & XY & 493 & 1 & 1 & 0 & 5 \\
6 & 2,643 & Yes & XX & XY & 494 & 0 & 0 & 0 & 0 \\
7 & 4,192 & Yes & XX & XY & 474 & 8 & 0 & 0 & 0 \\
\hline
\end{tabular}


resident or whether they require continual replenishment from the bone marrow. Two features of our current study allow us to conclude that the BAL-derived MSC population described herein resides locally within the human adult human lung: (a) the ability to study these cells from sex-mismatched lung transplant recipients; and (b) the ability to isolate (disaggregated) cells in ex vivo tissue culture at the single-cell level and analyze sex chromosome status in a quantitative manner. Our studies show that the vast majority of lung-derived MSCs are donor derived and, hence, resident in the lung at least from the time that the allograft was first transplanted. In 5 of 7 sex-mismatched lung transplants, no cells with the sex genotype of the recipient were seen. Although polyploidy suggestive of fusion events was noted in 4 of these cases, the rate of polyploidy was relatively low, ranging from $1.2 \%$ to $5.6 \%$. Interestingly, in almost all cases where such fusion events were noted, they occurred between 2 donor cells, and not between donor and recipient cells. Of a total of 3,500 cells analyzed (500 cells in each sex-mismatched patient), only 8 cells $(0.2 \%)$ with the same sex chromosome as that of the host were identified, suggesting that extrapulmonary or bone marrow contribution to this MSC-like cell population is extremely limited. It is intriguing that 7 of these 8 cells were found in the patient with the longest interval from lung transplantation (11.5 years); however, the patient with the next-longest interval from transplantation (7.2 years) was found to have no cells of host or recipient origin. Studies in larger numbers of long-surviving lung transplant recipients are needed to definitively assess the potential replacement of tissueresident MSC-like cells that might occur over more extended time periods ( $>7$ years).

Previous studies demonstrating engraftment of nonhematopoietic tissues by hematopoietic progenitor/stem cells in animal models and human studies in allogeneic sex-mismatched organ transplantations have relied on in situ labeling and immunophenotyping $(24,25)$. A limitation of these studies has been the inability to exclude recipient bone marrow-derived circulating cells located in close proximity to structural tissue-specific cells, or cell fusion events (24). A major advantage of the experimental approach used in our studies is that we were able to study these cells at the single-cell level; therefore, potential confounding factors such as adjacent/overlying bone marrow-derived or circulating immune cells were avoided. An inherent disadvantage of this approach, however, is that the in situ microenvironmental niche of these MSCs within the lung is currently not known. However, the derivation of allograft-specific MSCs definitively supports the presence of a lung-resident MSC population. This lung-resident MSC population is likely to lay dormant in the uninjured adult lung and become mobilized during states of lung repair/regeneration. In support of this concept, time from transplantation was predictive of whether MSC recovery/growth as assessed by BAL was successful $(1.27 \pm 2.01$ years with recovery versus $2.3 \pm 3.56$ years with no recovery; $P=0.007)$. The degree of severity of injury to the airway-alveolar epithelium is likely to have been highest during the immediate posttransplantation period, thus resulting in higher rates of MSC isolation. We have previously reported the ability to isolate alveolar mesenchymal cells from patients with acute lung injury with high rates of recovery, although the multipotential nature and origin(s) of these cells have not been determined (26). Furthermore, in the current study, although MSCs could be isolated from BAL samples from normal healthy volunteers, the rate of successful isolation was relatively low.
The donor derivation of MSCs from allografts of longer-surviving lung transplant recipients provides another important insight into mechanisms of connective tissue repair in the adult lung. In 4 of our sex-mismatched lung transplant recipients, several years (i.e., 3.1-11.5) transpired between the time of original lung transplantation and isolation of BAL-derived MSCs. Each of these patients had BOS, suggesting that their grafts had been subjected to immunological, and possibly nonimmunological, injury. Despite this chronic injury, MSCs isolated from these grafts were predominantly donor in origin. This suggests that these MSCs survive for long intervals within the lung or, more likely, that they possess self-renewal capacity. It is not known whether these cells in the postnatal human adult lung are present from the time of birth. In animal studies, a population of resident cells (side population cells; $\mathrm{CD} 45^{-} \mathrm{CD} 31^{-}$), potentially representing smooth muscle precursors, has been identified in murine embryonic lung (27).

Bone marrow-derived MSCs play an important role in supporting hematopoiesis by providing stromal support and by secretion of cytokines and growth factors $(28,29)$. One would predict that MSCs in different tissue microenvironments are phenotypically distinct to support organ-specific functions. In our analyses, gene expression profiling demonstrated significantly decreased expression in lung-derived MSCs of several factors implicated in hematopoiesis. Similarly, baseline expression of osteopontin, a marker for osteogenesis and an important factor in hematopoiesis, was significantly higher in bone marrow-derived MSCs than BALderived MSCs. These data support the presence of a unique lungresident MSC population.

The study of stem/precursor cells in the lung is an area of active research (30). Lung-specific epithelial cell stem/progenitor cells are known to be present along the respiratory tree (31-33), although bona fide MSCs resident in the lung have not been previously identified, to our knowledge, in humans. There is significant interest in the role of bone marrow-derived mesenchymal precursor cells, both MSCs (34) and a CD34+collagen $1^{+}$population termed fibrocytes $(14,35,36)$, in murine models of lung injury. However, the role of these bone marrow-derived cells in lung injury/repair, particularly in humans, is unclear (37). It is possible that specific bone marrow-derived populations such as circulating fibrocytes require specific microenvironmental signals and may not survive/grow in the ex vivo cell culture conditions used in our studies; thus, their participation, either directly or indirectly (e.g., by release of soluble factors), in reparative processes in lung transplantation cannot be excluded.

In summary, this study demonstrates, for the first time to our knowledge, the isolation and characterization of a nonhematopoietic MSC population from the lower respiratory tract of human lung transplant recipients. The ability to isolate these donorderived cells as long as 11.5 years after transplantation suggests the existence of a population of MSCs that reside and self-renew in the adult lung - a paradigm that might hold true for other organ systems as well. Identification and characterization of these MSCs could represent the first step to understanding endogenous mechanisms of adaptive and maladaptive lung injury repair.

\section{Methods}

Patients and samples. Lung transplant recipients undergoing bronchoscopy at the University of Michigan were eligible for enrollment in the study. The study was approved by the University of Michigan Institutional Review Board, and informed consent was obtained prior to participation in the 
study. Bronchoscopy and BAL with and without transbronchial biopsies using standard techniques were performed on all enrolled patients. BAL fluid was allocated for clinical tests, including bacterial, fungal, and viral cultures, and an additional $10-50 \mathrm{ml}$ of the BAL fluid was immediately placed on ice and processed for isolation of mesenchymal cells. Transbronchial biopsies were examined for the presence of acute rejection or bronchiolitis obliterans according to established criteria (38). BOS was defined by physiological testing according to the International Society of Heart and Lung Transplantation guidelines (39).

Isolation and culture of cells from BAL fluid. Recovered BAL fluid was initially filtered through sterile gauze to remove noncellular particulate material and mucus. Cells were pelleted by centrifugation at $1,000 \mathrm{~g}$ for 5 minutes and seeded at a density of $2 \times 10^{6}$ mononuclear cells per $100-\mathrm{mm}$ cell culture dish. The cells were maintained in medium consisting of high-glucose DMEM supplemented with $10 \%$ fetal bovine serum (FBS) (Invitrogen), $100 \mathrm{U} / \mathrm{ml}$ penicillin/streptomycin (Invitrogen), and $0.5 \%$ fungizone (Invitrogen) and incubated at $37^{\circ} \mathrm{C}$ in $5 \% \mathrm{CO}_{2} / 95 \%$ air. Medium was changed first after 24 hours and then every 3 days. Single separated fibroblastoid colonies termed CFU-Fs were identified at a mean interval of 14 days (range, 7-21 days) after initial plating. To study mesenchymal cells obtained from an individual patient, all colonies growing in a 100 -cc plate were trypsinized and passed into a T-75 flask. A homogeneous population of mesenchymal cells was obtained from individual patients by second passage. To study clonally derived cell populations, individual CFU-Fs were isolated with a sterile pipette and transferred to a T-25 flask. For staining and enumeration of CFU-Fs, adherent CFU-F colonies were fixed with methanol and stained with Wright-Giemsa stain (Fisher Scientific).

Immunofluorescence labeling. Immunofluorescence labeling was performed on 5 cell lines generated from individual patients at passages 2-6 using mouse mAbs against Ki67 (Novus Biologicals), prolyl-4-hydroxylase (Dako), and vimentin (Sigma-Aldrich). Cells were visualized and photographed using a Zeiss fluorescence microscope.

Multiparameterflow cytometric analyses (FACS). Cell-surface antigen phenotyping was performed on cell lines $(n=6-11)$ generated from individual patients at passage 3-5 using FITC- or PE-conjugated antibodies against CD34, CD45, CD14, CD73, CD90, and CD105 (all from SouthernBiotech). FITC-labeled antibodies against HLA class I antigen and HLA-DQ and PE-labeled antibody against HLA-DR were obtained from Sigma-Aldrich. Briefly, cells were trypsinized and aliquoted at a concentration of $0.5 \times 10^{6} \mathrm{cells} / \mathrm{ml}$ and stained for 30 minutes with either conjugated specific antibodies or isotype-matched control mouse IgGs at recommended concentrations. Labeled cells were washed twice, resuspended in FACS buffer, and analyzed on a FACSCalibur E5060 flow cytometer using FACSComp version 4.2 software (BD).

Assays for adipogenic, chondrogenic, and osteogenic differentiation. Multilineage differentiation to adipocyte, chondrocyte, and osteocyte lineages was tested in 15 different cell lines generated from individual patients at varying passage numbers (passages 2-6). Furthermore, to demonstrate the ability of cells derived from single cells to differentiate into multiple lineages, individual CFU-Fs were isolated and cultured, and differentiation of expanded cells from individual CFU-Fs $(n=10)$ was studied at the fourth passage. Induction conditions used were based on previous literature in the MSC field $(1,10,11)$. Briefly, for adipogenic differentiation, confluent cell cultures in a 24 -well plate were treated with adipogenic differentiation medium containing $0.5 \mathrm{mM}$ isobutylmethylxanthine, $200 \mu \mathrm{M}$ indomethacin, $10^{-6} \mathrm{M}$ dexamethasone, and $10 \mu \mathrm{g} / \mathrm{ml}$ of insulin in high-glucose DMEM (Invitrogen) with 10\% FBS (0.5 ml/well). At 3 weeks cells were fixed with $10 \%$ formaldehyde (Fisher Scientific) and incubated with fresh oil red $\mathrm{O}$ for 1 hour at room temperature; lipid droplets were visualized and photographed. Osteogenic differentiation was induced by incubating the cells with $10^{-8} \mathrm{M}$ dexamethasone, $0.2 \mathrm{mM}$ ascorbate phos- phate, and $10 \mathrm{mM} \beta$-glycerophosphate in $\alpha$-MEM (Invitrogen) with $10 \%$ FBS. After 21 days, cells were fixed and incubated with freshly made $2 \%$ alizarin red stain, $\mathrm{pH} 4$.2, for 3 minutes. For chondrogenic differentiation, cells were cultured as a pelleted micromass in the presence of TGF- $\beta_{3}$, as described by Pittenger et al. (1). After 4 weeks, pellets were embedded en bloc in Tissue Freezing Medium (TBS) in a cryomold in liquid nitrogen, and $6-\mu \mathrm{M}$ frozen sections were obtained. Immunohistochemical staining for the extracellular matrix proteoglycan aggrecan using anti-human aggrecan polyclonal antibody ( $1 \mu \mathrm{g} / 100 \mu \mathrm{l}$; R\&D Systems) was performed to demonstrate chondrocyte differentiation. All reagents, unless otherwise specified, were from Sigma-Aldrich.

RNA isolation and analysis. Total RNA was isolated from mesenchymal cells using the RNeasy Mini Kit (QIAGEN) as per the manufacturer's instructions. RT-PCR reaction was performed for expression of PPAR $\gamma 2$, aP2, type II collagen, type IX collagen, and osteopontin. $\beta_{2}$-Microglobulin was used as a control. Reverse transcription and PCR were carried out using a QIAGEN 1-step RT-PCR kit as per the manufacturer's protocol. The PCR reactions were carried out for 40 cycles with the primer sequences as described by Pittenger et al. (1). The reaction products were resolved by electrophoresis on a $1.0 \%$ agarose gel and visualized with ethidium bromide.

Real-time quantitative PCR analysis was performed on an ABI Prism 7000 SDS (Applied Biosystems) using the TaqMan PCR Master Mix (Applied Biosystems). The TaqMan real-time PCR primers were purchased from Applied Biosystems and included Hs00234592_m1 for PPAR- $\gamma$, Hs00609791_m1 for FABP4, Hs00758162_m1 for ALPL (alkaline phosphatase liver/bone/kidney), Hs00167093_m1 for SPP1 (secreted phosphoprotein 1, osteopontin), and Hs00202971_m1 for AGC1 (aggrecan 1).

For Affymetrix analysis, MSCs were isolated from the normal human bone marrow aspirate as previously described (40) under a protocol approved by the University of Michigan Institutional Review Board. Total RNA was prepared from 3 sets each of bone marrow- and BAL-derived MSCs cultured under similar conditions at passage 3. Affymetrix array hybridization and scanning were performed by the UMCCC Affymetrix and cDNA Microarray Core Facility at the University of Michigan, using Human U133 Plus 2.0 chips. Expression values for each gene were calculated using a robust multiarray average algorithm and expressed as $\log _{2}$-transformed data. Twotailed Student's $t$ test was used to determine differential expression of genes between lung-derived and bone marrow-derived MSCs.

FISH analysis of BAL-derived cells. Cultured cells from 7 patients were trypsinized, resuspended in AmnioMax culture medium and supplement (Invitrogen), and subcultured on 4 coverslips for 1-3 days to obtain actively dividing cells for cytogenetic analysis. Next, cells were harvested using a Tecan RSP 500 Robotic Harvester following a modified harvesting technique (41). CEP X Spectrum Orange and Y Spectrum Green DNA probes (Vysis) were hybridized to the cultured cells according to the manufacturer's instructions. These probes hybridize to the $\alpha$ satellite sequences in the centromeric region of the X chromosome and the Yq12 satellite III DNA region of the $\mathrm{Y}$ chromosome. A total of 500 interphase cells was scored for each of the 7 sex-mismatched cell lines studied; cells were also analyzed in metaphase for probe confirmation. The analysis was performed by a cytogeneticist who was blinded to donor and recipient sex. Normal male and female control slides were included in the FISH analysis to confirm validity of the probes.

Statistics. Generalized estimating equations (19) were used to model the potentially correlated binary outcomes of ability or inability to identify CFU-Fs in BAL samples. This standard statistical method for analyses of longitudinal data was used because multiple BAL samples were obtained from some patients, and this method allowed us to account for the dependence between sample outcomes on a given patient. Both univariate and multivariate analysis adjusting for potential confounders were studied. $P$ values of less than 0.05 were considered significant. 


\section{Acknowledgments}

Supported in part by NIH grants K23 HL077719 (to V.N. Lama), P50 HL074024 (to V.J. Thannickal), R01 HL067967 (to V.J. Thannickal), P50HL56402 (to M. Peters-Golden, G.B. Toews, and F.J. Martinez), R01 HL55397 (to D.J. Pinsky), and R01 HL085149 (to D.J. Pinsky) and a grant from the Scleroderma Research Foundation (to V.N. Lama and D.J. Pinsky).

1. Pittenger, M.F., et al. 1999. Multilineage potential of adult human mesenchymal stem cells. Science. 284:143-147.

2. Gerson, S.L. 1999. Mesenchymal stem cells: no longer second class marrow citizens. Nat. Med. 5:262-264.

3. Pereira, R.F., et al. 1995. Cultured adherent cells from marrow can serve as long-lasting precursor cells for bone, cartilage, and lung in irradiated mice. Proc. Natl. Acad. Sci. U. S. A. 92:4857-4861.

4. Prockop, D.J. 1997. Marrow stromal cells as stem cells for nonhematopoietic tissues. Science. 276:71-74.

5. Zvaifler, N.J., et al. 2000. Mesenchymal precursor cells in the blood of normal individuals. Arthritis Res. 2:477-488.

6. De Bari, C., Dell'Accio, F., Tylzanowski, P., and Luyten, F.P. 2001. Multipotent mesenchymal stem cells from adult human synovial membrane. Arthritis Rheum. 44:1928-1942.

7.Zuk, P.A., et al. 2001. Multilineage cells from human adipose tissue: implications for cell-based therapies. Tissue Eng. 7:211-228.

8. Noth, U., et al. 2002. Multilineage mesenchymal differentiation potential of human trabecular bone-derived cells. J. Orthop. Res. 20:1060-1069.

9. Young, H.E., et al. 2001. Human reserve pluripotent mesenchymal stem cells are present in the connective tissues of skeletal muscle and dermis derived from fetal, adult, and geriatric donors. Anat. Rec. 264:51-62.

10. Pierdomenico, L., et al. 2005. Multipotent mesenchymal stem cells with immunosuppressive activity can be easily isolated from dental pulp. Transplantation. 80:836-842.

11. Sabatini, F., et al. 2005. Human bronchial fibroblasts exhibit a mesenchymal stem cell phenotype and multilineage differentiating potentialities. Lab. Invest. 85:962-971.

12. Epperly, M.W., Guo, H., Gretton, J.E., and Greenberger, J.S. 2003. Bone marrow origin of myofibroblasts in irradiation pulmonary fibrosis. Am.J. Respir. Cell Mol. Biol. 29:213-224.

13. Fine, A. 2004. Marrow cells as progenitors of lung tissue. Blood Cells Mol. Dis. 32:95-96.

14. Hashimoto, N., Jin, H., Liu, T., Chensue, S.W., and Phan, S.H. 2004. Bone marrow-derived progenitor cells in pulmonary fibrosis. J. Clin. Invest. 113:243-252.

Received for publication July 14, 2006, and accepted in revised form January 10, 2007.

Address correspondence to: Vibha N. Lama, Division of Pulmonary and Critical Care Medicine, Department of Internal Medicine, University of Michigan Health System, 1500 E. Medical Center Drive, 3916 Taubman Center, Ann Arbor, Michigan 48109-0360, USA. Phone: (734) 936-5201; Fax: (734) 936-8266; E-mail: vlama@umich.edu.

doi:10.1172/JCI200418847.

15. Arcasoy, S.M., and Kotloff, R.M. 1999. Lung transplantation. N. Engl.J. Med. 340:1081-1091.

16. Estenne, M., and Hertz, M.I. 2002. Bronchiolitis obliterans after human lung transplantation. Am. J. Respir. Crit. Care Med. 166:440-444.

17. Friedenstein, A.J., Chailakhjan, R.K., and Lalykina, K.S. 1970. The development of fibroblast colonies in monolayer cultures of guinea-pig bone marrow and spleen cells. Cell Tissue Kinet. 3:393-403.

18. Short, B., Brouard, N., Occhiodoro-Scott, T., Ramakrishnan, A., and Simmons, P.J. 2003. Mesenchymal stem cells. Arch. Med. Res. 34:565-571.

19. Liang, K., and Zeger, S.L. 1986. Longitudinal data analysis using generalized linear models. Biometrika. 73:13-22.

20. Majumdar, M.K., Thiede, M.A., Mosca, J.D., Moorman, M., and Gerson, S.L. 1998. Phenotypic and functional comparison of cultures of marrow-derived mesenchymal stem cells (MSCs) and stromal cells. J. Cell. Physiol. 176:57-66.

21. Tremain, N., et al. 2001. MicroSAGE analysis of 2,353 expressed genes in a single cell-derived colony of undifferentiated human mesenchymal stem cells reveals mRNAs of multiple cell lineages. Stem Cells. 19:408-418.

22. Jia, L., et al. 2002. Gene expression profile of human bone marrow stromal cells: high-throughput expressed sequence tag sequencing analysis. Genomics. 79:7-17.

23. Kim, C.G., et al. 2006. Profiling of differentially expressed genes in human stem cells by cDNA microarray. Mol. Cells. 21:343-355.

24. Kotton, D.N., Fabian, A.J., and Mulligan, R.C. 2005 Failure of bone marrow to reconstitute lung epithelium. Am. J. Respir. Cell Mol. Biol. 33:328-334.

25. Korbling, M., and Estrov, Z. 2003. Adult stem cells for tissue repair - a new therapeutic concept? N. Engl. J. Med. 349:570-582.

26. Horowitz, J.C., et al. 2005. Constitutive activation of pro-survival signaling in alveolar mesenchymal cells isolated from patients with nonresolving acute respiratory distress syndrome. Am. J. Physiol. Lung Cell. Mol. Physiol. 290: L415-L425.

27. Summer, R., et al. 2005. Embryonic lung side population cells are hematopoietic and vascular precur- sors. Am. J. Respir. Cell Mol. Biol. 33:32-40.

28. Dazzi, F., Ramasamy, R., Glennie, S., Jones, S.P., and Roberts, I. 2006. The role of mesenchymal stem cells in haemopoiesis. Blood Rev. 20:161-171.

29. Caplan, A.I., and Dennis, J.E. 2006. Mesenchymal stem cells as trophic mediators. J. Cell. Biochem. 98:1076-1084.

30. Weiss, D.J., et al. 2006. Adult stem cells, lung biology, and lung disease. Proc. Am. Thorac. Soc. 3:193-207.

31. Hogan, B.L. 1999. Morphogenesis. Cell. 96:225-233.

32. Kim, C.F., et al. 2005. Identification of bronchoalveolar stem cells in normal lung and lung cancer. Cell. 121:823-835.

33. Aliotta, J.M., et al. 2005. Stem cells and pulmonary metamorphosis: new concepts in repair and regeneration. J. Cell. Physiol. 204:725-741.

34. Ortiz, L.A., et al. 2003. Mesenchymal stem cell engraftment in lung is enhanced in response to bleomycin exposure and ameliorates its fibrotic effects. Proc. Natl. Acad. Sci. U. S. A. 100:8407-8411.

35. Bucala, R., Spiegel, L.A., Chesney, J., Hogan, M., and Cerami, A. 1994. Circulating fibrocytes define a new leukocyte subpopulation that mediates tissue repair. Mol. Med. 1:71-81.

36. Moore, B.B., et al. 2005. CCR2-mediated recruitment of fibrocytes to the alveolar space after fibrotic injury. Am. J. Pathol. 166:675-684.

37. Lama, V.N., and Phan, S.H. 2006. The extrapulmonary origin of fibroblasts: stem/progenitor cells and beyond. Proc. Am. Thorac. Soc. 3:373-376.

38. Yousem, S.A., et al. 1996. Revision of the 1990 working formulation for the classification of pulmonary allograft rejection: Lung Rejection Study Group. J. Heart Lung Transplant. 15:1-15.

39. Estenne, M., et al. 2002. Bronchiolitis obliterans syndrome 2001: an update of the diagnostic criteria. J. Heart Lung Transplant. 21:297-310.

40. Krebsbach, P.H., et al. 1997. Bone formation in vivo: comparison of osteogenesis by transplanted mouse and human marrow stromal fibroblasts. Transplantation. 63:1059-1069.

41. Barch, M., Knutsen, T., and Spurbeck, J. 1997. Prenatal chromosome diagnosis. In The AGT cytogenetics laboratory manual. Lippincott-Raven. Philadelphia, Pennsylvania, USA. 229-233. 